\title{
HIV-1 subtype distribution in the Gambia and the significant presence of CRF49_cpx, a novel circulating recombinant form
}

\author{
Thushan I de Silva ${ }^{1,2}$, Roxanne Turner ${ }^{1}$, Stéphane Hué ${ }^{2}$, Roochi Trikha', Carla van Tienen ${ }^{1}$, Clayton Onyango ${ }^{1}$,
} Assan Jaye ${ }^{1}$, Brian Foley ${ }^{4}$, Hilton Whittle ${ }^{1}$, Sarah L Rowland-Jones ${ }^{3}$, Matthew Cotten ${ }^{1 *}$

\begin{abstract}
Background: Detailed local HIV-1 sequence data are essential for monitoring the HIV epidemic, for maintaining sensitive sequence-based diagnostics, and to aid in designing vaccines.

Results: Reported here are full envelope sequences derived from 38 randomly selected HIV-1 infections identified at a Gambian clinic between 1991 and 2009. Special care was taken to generate sequences from circulating viral RNA as uncloned products, either by limiting dilution or single genome amplification polymerase chain reaction (PCR). Within these 38 isolates, eight were subtyped as A and 18 as CRF02_AG. A small number of subtype B, C, D viruses were identified. Surprising, however, was the identification of six isolates with subtype J-like envelopes, a subtype found normally in Central Africa and the Democratic Republic of the Congo (DRC), with gag p24 regions that clustered with subtype A sequences. Near full-length sequence from three of these isolates confirmed that these represent a novel circulating recombinant form of HIV-1, now named CRF49_cpx.

Conclusions: This study expands the HIV-1 sequence database from the Gambia and will provide important data for HIV diagnostics, patient care, and vaccine development.
\end{abstract}

\section{Background}

Current data on the HIV epidemic in the Gambia are lacking. The most recent published data on HIV prevalence in the general population are from a nationwide perinatal clinic survey in 2000-2001 and indicate a low, but possibly increasing prevalence of HIV-1 infection in the country [1]. More recent data from the Medical Research Council Laboratories Genitourinary medicine (GUM) clinic indicate that although HIV-2 infection frequency is declining in patients attending the clinic, the HIV-1 prevalence rose from $4.2 \%$ in 1988 to $17.5 \%$ in 2003 [2]. Information on the genetic diversity of the local HIV-1 subtypes and genetic variety is also not abundant. The Los Alamos HIV Database (LAHDB) [3] currently lists only 31 sequence entries reporting subtype information from the Gambia, while the surrounding country Senegal has 840 reports, neighboring Mali

\footnotetext{
* Correspondence: matt.cotten@web.de

'Medical Research Council (UK) Laboratories, Atlantic Road, PO Box 273,

Fajara, The Gambia

Full list of author information is available at the end of the article
}

has 392, and Guinea Bissau has 290. Detailed sequence data are required to correctly document the AIDS epidemic, to trace the infection history, monitor changes in infection patterns and to maintain sensitive and accurate viral diagnostics. Furthermore, whether future HIV-1 vaccine strategy is based on immunogens optimized for local strains, or recently described 'global' mosaic vaccines that maximize coverage across HIV-1 strains worldwide $[4,5]$, ongoing documentation of HIV-1 sequence diversity is crucial. The current study was an attempt to improve the local HIV-1 sequence database.

Reported here are the full envelope gene (env) sequences derived from 38 HIV-1 infections identified at a Gambian clinic between 1991 and 2009, as well as three near full-genome sequences from a novel complex circulating recombinant form (CRF) identified in the study. The length of env sequence derived from each patient (approximately $2500 \mathrm{bp}$ ) allowed a robust determination of HIV-1 subtype. 


\section{Methods}

\section{Patient selection}

The viral sequences were obtained from patients attending the Genito-Urinary Medicine (GUM) clinic in Fajara, the Gambia, who had archived plasma samples available. Patient selection was based on two criteria (see below) and PCR was attempted on a total of 53 patient samples: the first group of 33 patients were selected at random from all those enrolled in the cohort with a CD4 count of $\geq 28 \%$ at diagnosis (these criteria were applied in order to use the amplified products for a concurrent study). The second group of five patients were selected at random from individuals who had recently been diagnosed with advanced HIV infection and started on antiretroviral therapy (ART); these patients therefore had lower CD4 counts (median CD4\% of 13 for the ART group, 35 for the non-ART group). Additional patient details are given in Table 1. For this second group of patients, the last blood sample before initiating ART was used as the source of virus.

\section{Viral RNA Extraction}

Viral RNA was extracted from $200 \mu \mathrm{l}$ of plasma diluted in $800 \mu \mathrm{l}$ of RNase free water using the QIAamp Ultrasens Viral RNA Extraction Kit (QIAGEN) with final elution into $60 \mu \mathrm{l}$. Each sample was loaded on a single column and washed according to the manufacturer's protocol.

\section{Amplification of full-length HIV-1 env}

Reverse transcription and the first round of a nested PCR reaction were performed in single reaction. Each $25 \mu \mathrm{l}$ RT-PCR reaction contained the following mix: $1 \times$ PCR buffer Titan One Tube System (Roche Applied Science), $2.5 \mathrm{mM} \mathrm{MgCl} 2,400 \mathrm{nM}$ dNTP mix, $0.1 \mu \mathrm{M}$ of primers O_envf and O_envr, $0.208 \mathrm{U} / \mu \mathrm{l}$ RNase inhibitor, $1 \mu \mathrm{l}$ of the Titan One Tube enzyme mix and $5 \mu \mathrm{l}$ of extracted RNA. Reverse transcription proceed at $45^{\circ} \mathrm{C}$ for $45 \mathrm{~min}$. followed by $95^{\circ} \mathrm{C}$ for $3 \mathrm{~min}, 10$ cycles of $94^{\circ}$ $\mathrm{C}(30 \mathrm{sec}), 56^{\circ} \mathrm{C}(30 \mathrm{sec}), 68^{\circ} \mathrm{C}(3 \mathrm{~min})$, followed by 30 cycles of $\left.94^{\circ} \mathrm{C}(30 \mathrm{sec}), 56^{\circ} \mathrm{C} 30 \mathrm{sec}\right), 68^{\circ} \mathrm{C}(3 \mathrm{~min})$ plus $5 \mathrm{sec}$ time extension at $68^{\circ} \mathrm{C}$ after each round and a final extension of $7 \mathrm{~min}$ at $68^{\circ} \mathrm{C}$. The inner (nested) PCR reactions used $1 \mu \mathrm{l}$ of the first-round RT-PCR product in $50 \mu \mathrm{l}$ containing: $1 \times$ Buffer (with $1.5 \mathrm{mM}$ $\mathrm{MgCl}_{2}$ final concentration), 0.05U/ $\mu$ l Expand HiFi Plus polymerase (Roche Applied Science), $400 \mathrm{nM}$ dNTP mix, $0.25 \mu \mathrm{M}$ of primers MO130 and MO147. Amplification was conducted at $95^{\circ} \mathrm{C}$ for $3 \mathrm{~min}$ followed by 40 cycles of $94^{\circ} \mathrm{C}(15 \mathrm{sec}), 56^{\circ} \mathrm{C}(30 \mathrm{sec}), 72^{\circ} \mathrm{C}(3 \mathrm{~min})$, and a final extension of $7 \mathrm{~min}$ at $72^{\circ} \mathrm{C}$. The PCR products were resolved on a $1 \%$ agarose (Tris-Borate EDTA, TBE) gel, DNA was visualized by ethidium bromide
Table 1 Cohort Summary

\begin{tabular}{|c|c|c|c|c|c|c|}
\hline ID & Sex & $\begin{array}{c}\text { Age at } \\
\text { diagnosis }\end{array}$ & Ethnicity & ART & $\begin{array}{c}\text { Year of } \\
\text { diagnosis }\end{array}$ & Subtype \\
\hline N006909 & $\mathrm{F}$ & 27 & Wolof & no & 1997 & $A$ \\
\hline N009845 & $\mathrm{F}$ & 42 & Jola & no & 2000 & A \\
\hline N040736 & $\mathrm{F}$ & 29 & Mandinka & no & 2005 & A \\
\hline N057856 & $\mathrm{F}$ & 25 & Mandinka & no & 2009 & A \\
\hline N058579 & M & 49 & Mandinka & yes & 2009 & A \\
\hline N059096 & $\mathrm{F}$ & 35 & Jola & yes & 2009 & A \\
\hline N33456 & $M$ & 52 & Fula & no & 2005 & A \\
\hline N75698 & $\mathrm{F}$ & 50 & Manjago & no & 1994 & A \\
\hline N004445 & $M$ & 37 & Jola & no & 1999 & CRF02_AG \\
\hline N010897 & $M$ & 25 & Mandika & no & 1999 & CRF02_AG \\
\hline N011064 & $\mathrm{F}$ & 34 & Mandinka & no & 2000 & CRF02_AG \\
\hline N016805 & $\mathrm{F}$ & 35 & Jola & no & 2002 & CRF02_AG \\
\hline N017561 & $\mathrm{F}$ & 32 & Mandinka & no & 2002 & CRF02_AG \\
\hline N018622 & $M$ & 18 & Mandinka & no & 2000 & CRF02_AG \\
\hline N022314 & $\mathrm{F}$ & 32 & Mandinka & no & 2003 & CRF02_AG \\
\hline N041366 & M & 40 & Mandinka & no & 2006 & CRF02_AG \\
\hline N047046 & $F$ & 60 & Mandinka & no & 2006 & CRF02_AG \\
\hline N056537 & $\mathrm{F}$ & 24 & Mandinka & no & 2008 & CRF02_AG \\
\hline N058521 & $M$ & 64 & Wolof & no & 2008 & CRF02_AG \\
\hline N058628 & $F$ & 26 & Wolof & yes & 2009 & CRF02_AG \\
\hline N059677 & $\mathrm{F}$ & 30 & Other & yes & 2009 & CRF02_AG \\
\hline N180032 & $F$ & 30 & Fula & no & 1995 & CRF02_AG \\
\hline N32458 & $F$ & 24 & Mandinka & no & 2003 & CRF02_AG \\
\hline N32468 & $F$ & 26 & Wolof & no & 2004 & CRF02_AG \\
\hline N36165 & $F$ & 25 & Jola & no & 2005 & CRF02_AG \\
\hline N73487 & $\mathrm{F}$ & 25 & Fula & no & 1993 & CRF02_AG \\
\hline N059733 & $M$ & 39 & Wolof & yes & 2009 & B \\
\hline N005312 & $\mathrm{F}$ & 22 & Mandinka & no & 1991 & $C$ \\
\hline N025015 & $\mathrm{F}$ & 30 & Mandinka & no & 2003 & $C$ \\
\hline N025567 & M & 34 & Fula & no & 1996 & $C$ \\
\hline N001823 & $F$ & 20 & Jola & no & 1998 & D \\
\hline N73603 & $F$ & 23 & Serahuli & no & 1993 & D \\
\hline N001605 & $\mathrm{F}$ & 22 & Jola & no & 1998 & CRF49_cpx \\
\hline N005284 & $\mathrm{F}$ & 20 & Mandinka & no & 1999 & CRF49_cpx \\
\hline N018380 & $M$ & 29 & Manjago & no & 2002 & CRF49_cpx \\
\hline N024017 & $F$ & 29 & Mandinka & no & 1998 & CRF49_cpx \\
\hline N026677 & $\mathrm{F}$ & 37 & Manjago & no & 2002 & CRF49_cpx \\
\hline N28353 & $\mathrm{F}$ & 29 & Serahuli & no & 1996 & CRF49_cpx \\
\hline
\end{tabular}

staining and the $2.5 \mathrm{~kb}$ product purified using the MinElute Gel Extraction Kit (QIAGEN).

\section{Amplification of HIV-1 p24}

Reverse transcription and the first round of a nested PCR reaction were performed in single reaction. Each $50 \mu \mathrm{l}$ $\mathrm{RT}-\mathrm{PCR}$ reaction contained the following mix: $1 \times \mathrm{PCR}$ 
buffer Titan One Tube System (Roche Applied Science), $2.5 \mathrm{mM} \mathrm{MgCl}_{2}, 200 \mathrm{nM}$ dNTP mix, $0.5 \mu \mathrm{M}$ of primers MO042 or MO024 (alternate outer forward) and MO044, $0.208 \mathrm{U} / \mu \mathrm{l}$ RNase inhibitor, $1 \mu \mathrm{l}$ of the Titan One Tube enzyme mix and $10 \mu \mathrm{l}$ of extracted RNA. Reverse transcription proceed at $50^{\circ} \mathrm{C}$ for $30 \mathrm{~min}$, followed by $95^{\circ} \mathrm{C}$ for $3 \mathrm{~min}, 40 \mathrm{cycles}$ of $94^{\circ} \mathrm{C}(30 \mathrm{sec}), 54^{\circ} \mathrm{C}(30 \mathrm{sec}), 72^{\circ} \mathrm{C}$ $(1 \mathrm{~min})$ and a final extension of $7 \mathrm{~min}$ at $72^{\circ} \mathrm{C}$. The inner (nested) PCR reactions used $1 \mu \mathrm{l}$ of the first-round RT-PCR product in $50 \mu$ l containing: $1 \times$ Buffer (with 1.5 $\mathrm{mM} \mathrm{MgCl} 2$ final concentration), $0.05 \mathrm{U} / \mu \mathrm{l}$ Expand $\mathrm{HiFi}$ Plus polymerase (Roche Applied Science), $400 \mathrm{nM}$ dNTP mix, $0.5 \mu \mathrm{M}$ of primers MO043 and MO045. Amplification was conducted at $95^{\circ} \mathrm{C}$ for $3 \mathrm{~min}$ followed by 40 cycles of $94^{\circ} \mathrm{C}(30 \mathrm{sec}), 56^{\circ} \mathrm{C}(30 \mathrm{sec}), 72^{\circ} \mathrm{C}(1 \mathrm{~min})$, and a final extension of $7 \mathrm{~min}$ at $72^{\circ} \mathrm{C}$. The PCR products were resolved on a $1 \%$ agarose (Tris-Borate EDTA, TBE) gel, DNA was visualized by ethidium bromide staining and the product was purified using the MinElute Gel Extraction Kit (QIAGEN).

\section{Amplfication of near full-length HIV-1 genomes}

In addition to env and p24 fragments, near full-length genome sequence was obtained by amplifying three further fragments: (A) 5' LTR to gag p24, (B) gag p24 to $e n v$ and (C) env to 3' LTR. For fragment (A), reverse transcription and the first round of a nested PCR reaction were performed in single reaction. Each $25 \mu \mathrm{l}$ RT-PCR reaction contained the following mix: $1 \times$ PCR buffer Titan One Tube System (Roche Applied Science), $2.5 \mathrm{mM} \mathrm{MgCl} 2,400 \mathrm{nM}$ dNTP mix, $0.5 \mu \mathrm{M}$ of primers MO034 and MO191, $0.208 \mathrm{U} / \mu \mathrm{l}$ RNase inhibitor, $1 \mu \mathrm{l}$ of the Titan One Tube enzyme mix and $5 \mu \mathrm{l}$ of extracted RNA. Reverse transcription proceed at $50^{\circ} \mathrm{C}$ for $30 \mathrm{~min}$, followed by $95^{\circ} \mathrm{C}$ for $3 \mathrm{~min}, 40$ cycles of $94^{\circ} \mathrm{C}(30 \mathrm{sec})$, $54^{\circ} \mathrm{C}(30 \mathrm{sec}), 72^{\circ} \mathrm{C}(1 \mathrm{~min})$ and a final extension of $7 \mathrm{~min}$ at $72^{\circ} \mathrm{C}$. The inner (nested) PCR reactions used $1 \mu \mathrm{l}$ of the first-round RT-PCR product in $50 \mu$ containing: $1 \times$ Buffer (with $1.5 \mathrm{mM} \mathrm{MgCl} 2$ final concentration), $0.05 \mathrm{U} / \mu \mathrm{l}$ Expand HiFi Plus polymerase (Roche Applied Science), $400 \mathrm{nM}$ dNTP mix, $0.5 \mu \mathrm{M}$ of primers MO024 and MO192. Amplification was conducted at $95^{\circ} \mathrm{C}$ for $3 \mathrm{~min}$ followed by 40 cycles of $94^{\circ} \mathrm{C}$ (30 sec), $56^{\circ} \mathrm{C}$ $(30 \mathrm{sec}), 72^{\circ} \mathrm{C}(1 \mathrm{~min})$, and a final extension of $7 \mathrm{~min}$ at $72^{\circ} \mathrm{C}$. Fragment $(\mathrm{C})$ was amplfied with a nested PCR on products obtained with primers O_envf and O_envr as described above. The inner (nested) PCR reactions and conditions were identical to those used above for fragment (A), but using primers MO193 and MO194. For fragment (B), reverse transcription was performed in a $20 \mu \mathrm{l}$ reaction containing $1 \times$ Qiagen LongRange RT buffer, $1 \mathrm{mM}$ mix of each dNTP, $1 \mu \mathrm{M}$ of primer MO187, $0.04 \mathrm{U} / \mu \mathrm{l}$ RNase inhibitor, $1 \mu$ l LongRange Reverse Transcriptase (Qiagen) and $10 \mu \mathrm{l}$ of extracted RNA. Reactions were incubated at $42^{\circ} \mathrm{C}$ for 90 minutes followed by $85^{\circ} \mathrm{C}$ for 5 minutes. Each $50 \mu$ first round PCR contained the following: 1× Expand Long Template (Roche Applied Science) buffer 1 (with $1.75 \mathrm{mM} \mathrm{MgCl} 2$ final concentration), $400 \mathrm{nM} \mathrm{dNTP}$ mix, $0.3 \mu \mathrm{M}$ of primers MO186 and MO187, $0.75 \mu \mathrm{l}$ of the Expand Long Template enzyme mix and $5 \mu \mathrm{l}$ of cDNA template. PCR conditions were as follows: $94^{\circ} \mathrm{C}$ for $2 \mathrm{~min}, 10$ cycles of $94^{\circ} \mathrm{C}(10 \mathrm{sec}), 56^{\circ} \mathrm{C}$ (30 sec), $68^{\circ} \mathrm{C}(4 \mathrm{~min})$, followed by 30 cycles of $94^{\circ} \mathrm{C}$ (10 sec), $56^{\circ} \mathrm{C}(30 \mathrm{sec}), 68^{\circ} \mathrm{C}(4 \mathrm{~min})$ plus $20 \mathrm{sec}$ time extension at $68^{\circ} \mathrm{C}$ after each round and a final extension of $7 \mathrm{~min}$ at $68^{\circ} \mathrm{C}$. The inner (nested) PCR used $1 \mu \mathrm{l}$ of the first round PCR product in $50 \mu \mathrm{l}$ containing $1 \times$ Expand Long Template (Roche Applied Science) buffer 1 (with $1.75 \mathrm{mM} \mathrm{MgCl} 2$ final concentration), $400 \mathrm{nM}$ dNTP mix, $0.5 \mu \mathrm{M}$ of primers MO188 and MO189 and $0.75 \mu \mathrm{l}$ of the Expand Long Template enzyme mix. Amplification was conducted using the same conditions as described above for the first round PCR.

\section{Limiting dilution PCR and Single Genome Amplification}

All env fragments were initially amplified using bulk PCR conditions on undiluted template and sequencing was carried out as described below for the highly variable $\mathrm{V} 1 / \mathrm{V} 2$ region, followed by the entire env fragment if no double peaks were observed. In those samples showing multiple peaks in the V1/V2 region, the cDNA was then amplified using two different dilution methods in order to obtain amplification from single genomes. Both methods involved diluting the cDNA and running a standard PCR. First, three-fold limiting dilution of a single cDNA sample (reverse transcribed using the Titan One Tube RT-PCR reaction mix, for $45 \mathrm{~min}$ at $45^{\circ} \mathrm{C}$ ) was carried out (from 1:3 to 1:243), followed by the standard first round and nest PCR conditions as described above. The highest dilution at which the env fragment amplification was successful was chosen for sequencing. If the V1/V2 region still contained multiple sequences, single genome amplification was carried out with a modified protocol to that described in the literature [6]. Briefly, three-fold dilution of cDNA was carried out with nine replicates per dilution (starting at the highest dilution at which the single sample limiting dilution PCR was successful), followed by the standard first round and nest PCR conditions as described above. An amplified env from the dilution where only one or two replicates yielded a positive PCR reaction (i.e. $<30 \%$ of replicates positive [6]) was selected for sequencing and purified using the MinElute Gel Extraction Kit (QIAGEN).

\section{Sequencing strategy}

The full-length env products were sequenced using a set of overlapping reactions. The internal nested primers, MO130 and MO147, were used as the 5' most and 3' most 
Table 2 Primers used in this work

\begin{tabular}{|c|c|c|c|}
\hline Name & Function & Position in $\mathrm{HXB2}^{2}$ & Sequence $\left(5^{\prime}\right.$ to $\left.3^{\prime}\right)$ \\
\hline O_envf & env PCR OF ${ }^{1}$ & $5964-5984$ & TYTCCTATGGCAGGAAGAAGC \\
\hline O_envr & env PCR OR & $9096-9074$ & TAACCCWTCCAGTCCCCCCTITT \\
\hline $\mathrm{MO130}$ & env PCR IF & $6207-6228$ & GAGCAGAAGACAGTGGCAATGA \\
\hline $\mathrm{MO147}$ & env PCR IR & $8834-8810$ & CATCCMACTATRCTRCTIITTGACC \\
\hline $\mathrm{MO150}$ & env sequencing & $6976-6955$ & ATTCCATGTGTACYTTGTACTG \\
\hline MO151 & env sequencing & $6859-6880$ & CAATTCCCATACATTATTGTGC \\
\hline MO152 & env sequencing & 7668-7647 & CACTTCTCCAATTGTCCRTCAT \\
\hline MO153 & env sequencing & $7516-7537$ & GACAAGCAATGTATGCCCCTCC \\
\hline MO154 & env sequencing & $8241-8220$ & ACCAATTCCACAYACTTGCCCA \\
\hline MO155 & env sequencing & $8050-8072$ & CTGGAACKCTAGTTGGAGTAAT \\
\hline $\mathrm{MO042}$ & gag p24 OF-1 & 890-909 & TAGTATGGGCAAGCAGGGAG \\
\hline MO024 & gag p24 OF-2 & $508-527$ & AACCCACTGCTTAAGCCTCA \\
\hline $\mathrm{MO044}$ & gag p24 OR & $2272-2252$ & TGCCAAAGAGTGATTTGAGGG \\
\hline MO043 & gag p24 IF & 1048-1067 & TGYGTRCATCAAARGATAGA \\
\hline $\mathrm{MO045}$ & gag p24 IR & 2118-2101 & CCCCTTGYTGGAAGGCCA \\
\hline MO034 & 5' LTR to gag p24 OF & $478-479$ & TGAGCCTGGGAGCTCTCTG \\
\hline MO186 & p24 to env OF & $1958-1985$ & TTAARTGTTTCAACTGTGGCAAAGAAGA \\
\hline MO187 & p24 to env OR & $6420-6445$ & CAAGCATGKGTAGCCCAGAYATTATG \\
\hline MO188 & p24 to env IF & $2034-2060$ & ATGTGGGAARGARGGACACCAAATGAA \\
\hline MO189 & $\mathrm{p} 24$ to env $\mathbb{R}$ & $6335-6360$ & TCCACACAGGTACCCCATAATAGACT \\
\hline MO191 & 5' LTR to gag p24 OR & $832-859$ & AATGCTGWRAACATGGGTATTACTTCTG \\
\hline MO192 & 5' LTR to gag p24 IR & $786-814$ & TCTATTACTTTYACCCATGCATTTAAAGT \\
\hline MO193 & env to 3' LTR IF & $7922-7944$ & CAGACCCTTATCCCAAACCCAAC \\
\hline MO194 & env to $3^{\prime} \mathrm{LTR} I \mathrm{R}$ & $8606-8629$ & CCCCCCTITCTITTAAAAAGWRGC \\
\hline AJB-1R & p24 to env sequencing & $2239-2262$ & TATGGATTTTCAGGYCCAATTYTTG \\
\hline $\mathrm{AJB}-2 \mathrm{~F}$ & p24 to env sequencing & $2036-2058$ & GCCCAAARGTTAAACAATGGCCA \\
\hline AJB-3R & p24 to env sequencing & $2846-2871$ & TTCTGTATRTCATTGACAGTCCAGCT \\
\hline AJB-4F & p24 to env sequencing & $2741-2765$ & ACACCAGAYAARAARCATCAGAAAG \\
\hline AJB-5R & p24 to env sequencing & $3585-3610$ & GATTCCTAATGCATACTGTGAGTCTG \\
\hline AJB-6F & p24 to env sequencing & $3585-3610$ & CAGACTCACAGTATGCATTAGGAATC \\
\hline AJB-7R & p24 to env sequencing & $3722-3750$ & ACTAATTTATCTACTTGTTCATTTCCGCC \\
\hline AJB-8R & p24 to env sequencing & $4357-4383$ & ATGTCTAYTATTCTTTCCCCTGCACTG \\
\hline AJB-9F & p24 to env sequencing & $4196-4219$ & ATTCCCTACAATCCCCAAAGMCARG \\
\hline AJB-10F & p24 to env sequencing & $4609-4633$ & TGATTGTGTGGCARGTAGACAGGAT \\
\hline AJB-11R & p24 to env sequencing & $4830-4854$ & TCCATTCTATGGAGACYCCMTGACC \\
\hline AJB-12R & p24 to env sequencing & $5498-5521$ & TGCCATAGGARATGCCTAAGCCYTT \\
\hline AJB-13F & p24 to env sequencing & $5498-5521$ & AARGGCTTAGGCATYTCCTATGGCA \\
\hline
\end{tabular}

${ }^{1}$ Abbreviations:OF, outer forward; OR, outer reverse; IF, inner forward, IR, inner reverse.

${ }^{2}$ HXB2 numbering is based on sequence with accession number K03455.

primers for sequencing. An additional six primers were designed to generate eight contigs covering the full env sequence (see Table 2 for details). Sequencing primers were designed to hybridize to conserved regions ca. 600800 bp apart using a collection of 30 West African sequences from the LAHDB plus the reference HIV-1 HXB2. The p24 PCR products were sequenced using internal nested primers MO043 and MO045. Additional fragments required to assemble near full-genome sequence were sequenced as follows: fragments (A) and (C) were sequenced with internal nested primers MO024/MO192 and MO193/MO194 respectively. For fragment (B), internal nested primers, MO188 and MO189 were used as the 5' most and 3' most primers, along with 13 additional primers designed as described above to span the entire region from gag to env (see Table 2 for details). All primers for PCR and 
sequencing were synthesized by Metabion (Metabion International AG, Lena-Christ-Str. 44/I, 82152 Martinsried, Germany, [7]). Sequencing reactions were carried out by Macrogen [8].

\section{Assembling full-length env, p24 and near full-genome sequences}

For all samples, the sequencing chromatograms were carefully inspected for sites of ambiguous sequence. All reliable sequence data were assembled using the BioEdit Sequence Alignment Editor [9,10] and aligned using the Cap Contig Assembly program. For each assembled sequence, the open reading frame (ORF) was established using alignments with HXB2 env and the ORF finder in the Sequence Manipulation Suite [11,12]. In areas where premature stop codons appeared, the sequence chromatograms were re-examined to determine if miscalled nucleotides in the region could account for the loss of the open reading frame. Such errors were manually corrected to give full reads of the respective sequence.

All sequences described in this manuscript have been deposited in GenBank with the following accession numbers: Envelopes ( $\mathrm{n}=35$ ): HQ385442 - HQ385476; CRF49 genomes $(n=3)$ : HQ385477 - HQ385479; 3 extra p24 sequences from presumed CRF49 isolates (n = 3): HQ385480 - HQ385482.

\section{HIV-1 subtyping and phylogenetic analyses}

HIV-1 subtype was assigned to each completed sequence in the following manner. Env DNA sequences from each subject, along with the HIV-1 subtype reference set (2005) obtained from the LAHDB, additional CRF02_AG sequences DJ263 (Djibouti), MP1211 (Senegal), MP1213 (Senegal) (accession numbers AB485634, AJ251056 and AJ251057 respectively) and additional A3 env sequences from Senegal (DD1579, DDJ360, DDJ362 and DDJ364; accession numbers AY521629, AY521630, AY521632 and AY521633 respectively) $[13,14]$ were aligned using CLUSTALW2 [15,16]. All alignments were inspected and edited manually using Se-Al (Sequence Alignment editor, v2.0a11, Rambaut, A. Department of Zoology, University of Oxford, UK), and ambiguous regions with multiple indels were deleted. Phylogenetic trees were constructed with the program PAUP* version 4.0b10 [17] using a maximum likelihood (ML) approach [18]. The trees were reconstructed under the General Time Reversible model of nucleotide substitution [19], with proportion of invariable sites and substitution rate heterogeneity. The statistical robustness of the ML topologies was assessed by bootstrapping with 1000 replicates using the neighbour-joining method. The software Inkscape [20] was used to color code and label the trees.

\section{Phylogenies of env, p24 and near full-length sequences from CRF49_cpx isolates}

$E n v$ fragments from six individuals designated as subtype J-like using the phylogenetic analyses described above were further aligned with all available subtype J env sequence of approximately $1200 \mathrm{bp}$ or above in length in the LAHDB: SE92809 (AF082394), SE9173 (AF082395), MBTB4 (AJ401046), KTB147 (AJ401041), MBS41 (AJ4010145), VLGCJ1 (AY669766), VLGCJ2 (AY669767), 98BW21.17 (AF192135), GM4 (U33099), GMB22 (AJ276694) and GMB24 (AJ276695). All sequences were trimmed to the length of the shortest sequence, thus an alignment containing $1125 \mathrm{bp}$ fragments were used to build a subtype J env phylogenetic tree using the methodology described above.

The p24 sequence from these six individuals were also aligned with HIV-1 subtype A and CRF02_AG reference isolates from the LAHDB (2005) subtype reference set [3], additional gag sequence from three CRF02_AG isolates SE7812 (AF107770), MP1211 (AJ251056), MP1213 (AJ251057), three A3 Senegalese isolates DDJ360 (AY521630), DDI579 (AY521629), DDJ369 (AY521631) [13,14], additional subtype A1 isolates SE7535 (AF069671), SE8891 (AF069673), SE8131 (AF107771), SE8538 (AF069669). and the DRC isolates MBTB4 (AJ404293), KCC2 (AM000053), KTB13 (AM000054) and KTB035 (AM000055). A phylogenetic tree was reconstructed with the methodology described above.

Near full-genome sequences obtained from three of these isolates were aligned with the 2008 LAHDB subtype reference set and isolates 98 BW21.17 (AF192135), DDJ360 (AY521630), DDI579 (AY521629) and DDJ369 (AY521631). Bayesian Markov chain Monte Carlo (MCMC) phylogenies were estimated under the General Time Reversible model of nucleotide substitution with gamma-distributed rate heterogeneity, using the program MRBAYES version 3.1.2. [21]. The Bayesian MCMC search was set to $1,500,000$ iterations with trees sampled every 100 th generations. A maximum clade credibility tree (MCCT) was selected from the sampled posterior distribution with the programTreeAnnotator version 1.5.2 http://beast.bio.ed.ac.uk/, after discarding trees corresponding to a $10 \%$ burnin. The MCCT Tree was edited with the program FigTree version 1.1.2.

\section{Characterization of subtype recombination in CRF49_cpx}

Simplot and bootscan analyses of near full-genome isolates N18380_GM, N26677_GM and N28353_GM were performed using Simplot [22]. Pure subtypes A through $\mathrm{K}$ were included (and in a second analysis, isolate 98BW21.17 added) and the alignment was globally gap stripped. Sliding window was set to $400 \mathrm{bp}$ and increments set to $50 \mathrm{bp}$. Bootscanning was performed using 
the neighbour-joining method, using the Kimura (twoparameter) distance model and 100 bootstrap replicates for each sliding window. The transition/traversion ratio was set to 2.0. For each CRF49_cpx sequence, markers were placed at breakpoints between subtypes and an alignment of each fragment used to construct phylogenetic trees using the maximum likelihood methodology (and bootstrapping with 1000 replicates using the neighjour-joining method) described above. The HIV Sequence Locator tool at the LAHDB was used to assign HXB2 numbering to each fragment and the Recombinant HIV-1 Drawing Tool (also at the LAHDB) utilised to construct a recombinant map of CRF49_cpx representing a consensus of breakpoints across the three full genomes.

\section{Results and Discussion}

Description of the Cohort

The majority of the subjects was female ( $n=28,74 \%$ ); a higher percentage of women attending the GUM clinic in Gambia has been reported and may be due to changes in referral policies and sex-specific differences in health-care seeking behaviour [2]. The median age at diagnosis was 29.5 years. The ethnic composition of the cohort was largely similar to the Gambian general population with Mandinka 42\% (42\% in general population), Fula 11 (18), Wolof 13 (16), Jola 18 (10), Serahuli 5 (9), Manjago 8 (not listed) and other groups 3 (4). The numbers in parentheses are from the 2003 census data [23]. The number of Jola subjects (18.4\%) was noticeably higher than the general population (10\%).

\section{Virus subtyping}

The subtype assignment of the 38 env sequences was obtained by aligning the sequences with LAHDB HIV-1 (2005) subtype reference sequences (which includes approximately four reference sequences from each relevant subtype), along with an additional three CRF02_AG and four A3 sequences (two from A3/CRF02_AG recombinants) as described above and constructing a maximum likelihood tree. As none of the new Gambian env sequences clustered with currently known recombinant forms other than CRF02_AG, for clarity Fig. 1 displays reference isolates from pure subtypes and CRF02_AG only.

Five of the new Gambian sequences (N057856_GM, N059096_GM, N9845_GM, N75698_GM and N040736_GM) clustered with the Senegalese A3 (DDJ360, DD1579) and A3/CRF02_AG recombinant (DDJ364, DDJ362) sequences [13,14] with a bootstrap support of $81 \%$ (see Fig. 1 cluster denoted by $\infty$ ). Given the regional frequency of A3-like viruses, their occurrence in Gambia is not unexpected. Four isolates (N59677_GM, N058521_GM, N22314_GM and
N018622_GM) clustered with reference and Gambian CRF02_AG sequences (bootstrap support 83\%), although it can be difficult to distinguish subtype A (A1, A2, A3) from CRF02_AG isolates based in env alone as this region is largely subtype A derived in CRF02_AG [24]. An additional four isolates did not form significant clusters (N32458_GM, N47046_GM, N058628_GM and N006909_GM). Thus these data do not support the existence of a Gambian-specific AG sub-subtype. From this analysis, it appears that the heterogeneity within the global CRF02_AG subgroup is equally reflected within the Gambian AG viruses. It is clear that the subtype A env sequences from circulating Gambian strains are distinct from both A1 and A2 reference isolates in the $\mathrm{LAHDB}$, and more closely related to Senegalese A3 or CRF02_AG isolates.

In addition to the $\mathrm{A}$ and $\mathrm{AG}$ like isolates, the novel viruses include a single subtype B (N059733_GM), three subtype C isolates (N005312_GM, N25667_GM, N025015_GM) and two subtype D isolates (N73603_GM, N001823_GM) clustering with high bootstrap values within the reference isolate clusters for these subtypes (Fig. 1). Of special interest were six isolates (N18380_GM, N001605_GM, N24017_GM, N28353_GM, N005284_GM and N26677_GM) forming a monophyletic cluster within the subtype J branch (bootstrap value of $100 \%$, see Fig. 1 and below).

An additional consideration was raised by the recent analysis concluding that CRF02_AG is more likely to be a pure subtype and the precursor to subtype G, which may in turn be a recombinant derived from subtypes CRF02_AG and J [25]. This history could account for the high prevalence of CRF02_AG in West Africa and may account for local differences (for example between Senegal and Gambia) in the prevalence of subtype $G$ and J viruses. A more recent analysis has however questioned these claims and suggested that CRF02_AG did indeed arise as a result of recombination events that occurred early in the divergence between subtype A and G [26].

\section{Isolates with subtype J-like env have subtype A gag regions}

Three previous Gambian HIV-1 samples, GM4 (U33099), GM5 and GM7, were reported to be distinct from the pure HIV-1 subtypes A to G known at the time [27] when the J subtype had not yet been defined. GM4 is described in the LAHDB as a subtype CGJ mosaic, although phylogenetic analyses suggest that it is subtype J-like in env [28]. Since that time, two additional Gambian J-like env sequences were reported (GMB22, GMB24 [28]). GenBank was searched for sequences with genetic similarity to either the GMB22 or the N28353 sequences and additional subtype J env sequences were identified: VLGC-J1 (env from a virus identified in 


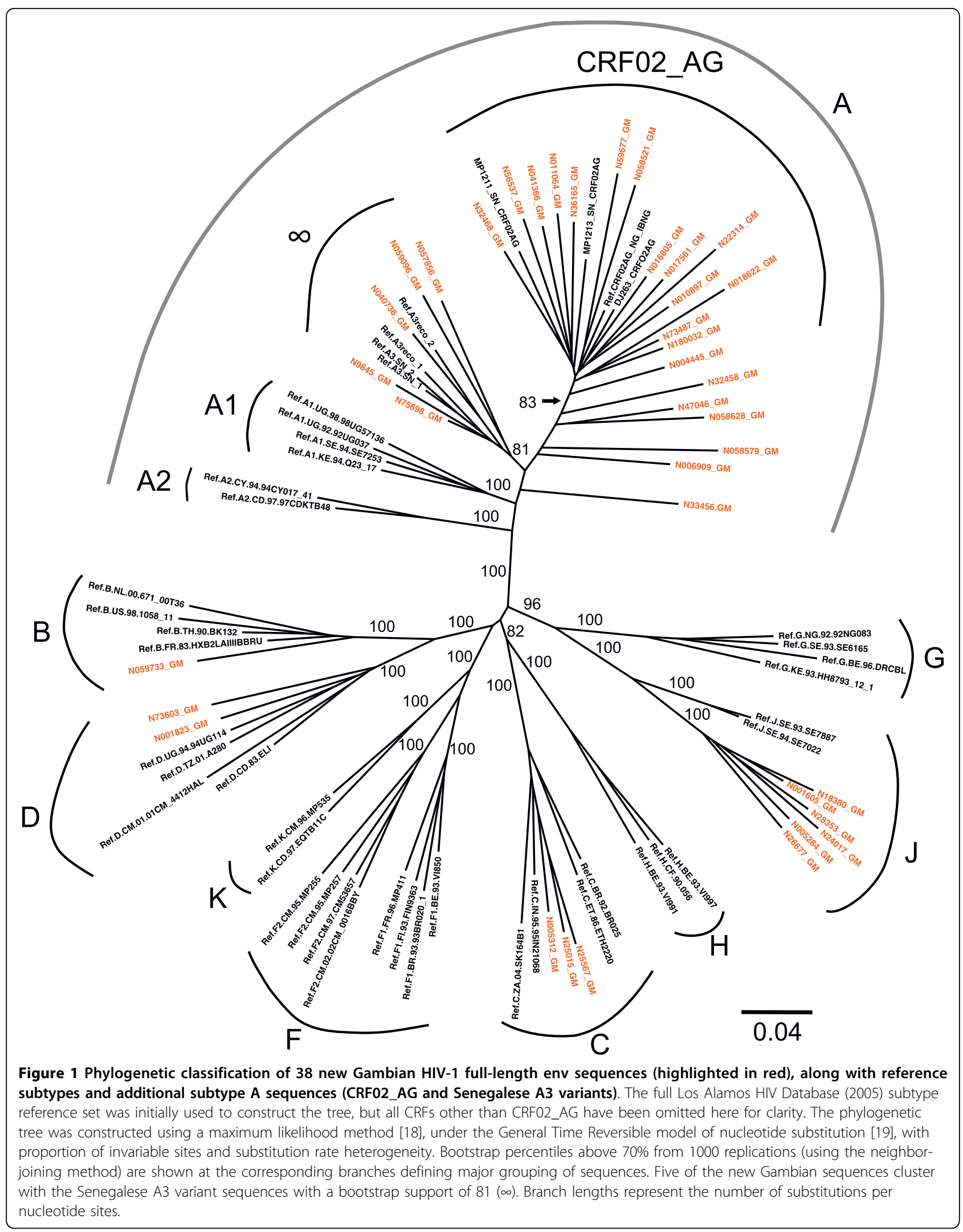


Germany), VLGC-J2 (of unknown origin) [29], the 98 BW21.17 isolate from Botswana [30] and the MBTB4, KTB147 and MBS41 isolates from DRC [31]. A phylogenetic tree was constructed as described above with these isolates, along with the six subtype J-like env samples from the current study (Fig. 2). All nine subtype J-like env sequences from the Gambia form a monophyletic cluster (with a bootstrap support of 92\%) and are distinct from the DRC isolates (Fig. 2).

The Botswana isolate was reported as a novel subtype A/J recombinant [30], although it has since been reclassified by the LAHDB as an AGJ recombinant, as parts of the genome are said to be more closely related to CRF06_AJGK than to any one isolate of subtype A or J [3]. The GMB22 and GMB24 isolates are also reported as having subtype A gag regions, although only gag sequence from GMB22 is available [28]. To test the idea that a novel recombinant is circulating in the Gambia, the gag p24 regions from the six novel J-like env isolates were sequenced and all were found to be subtype A. Furthermore the $\mathrm{gag}$ regions from the Botswana isolate 98BW21.17, GMB22 and five of the new A/J isolates form a monophyletic cluster with a bootstrap support of 94\% (Fig. 3). These gag isolates are distinct from subsubtype A1, A2, A3 sequences, as well as those derived from CRF02_AG isolates. One new recombinant isolate (N5284_GM) gag region clustered with A3 $[13,14]$ isolates reported in surrounding Senegal, which may indicate further recombination between the novel recombinant with circulating local A3 strains. One additional isolate described in the literature, MBTB4 from DRC, is reported to have a subtype A gag and subtype J

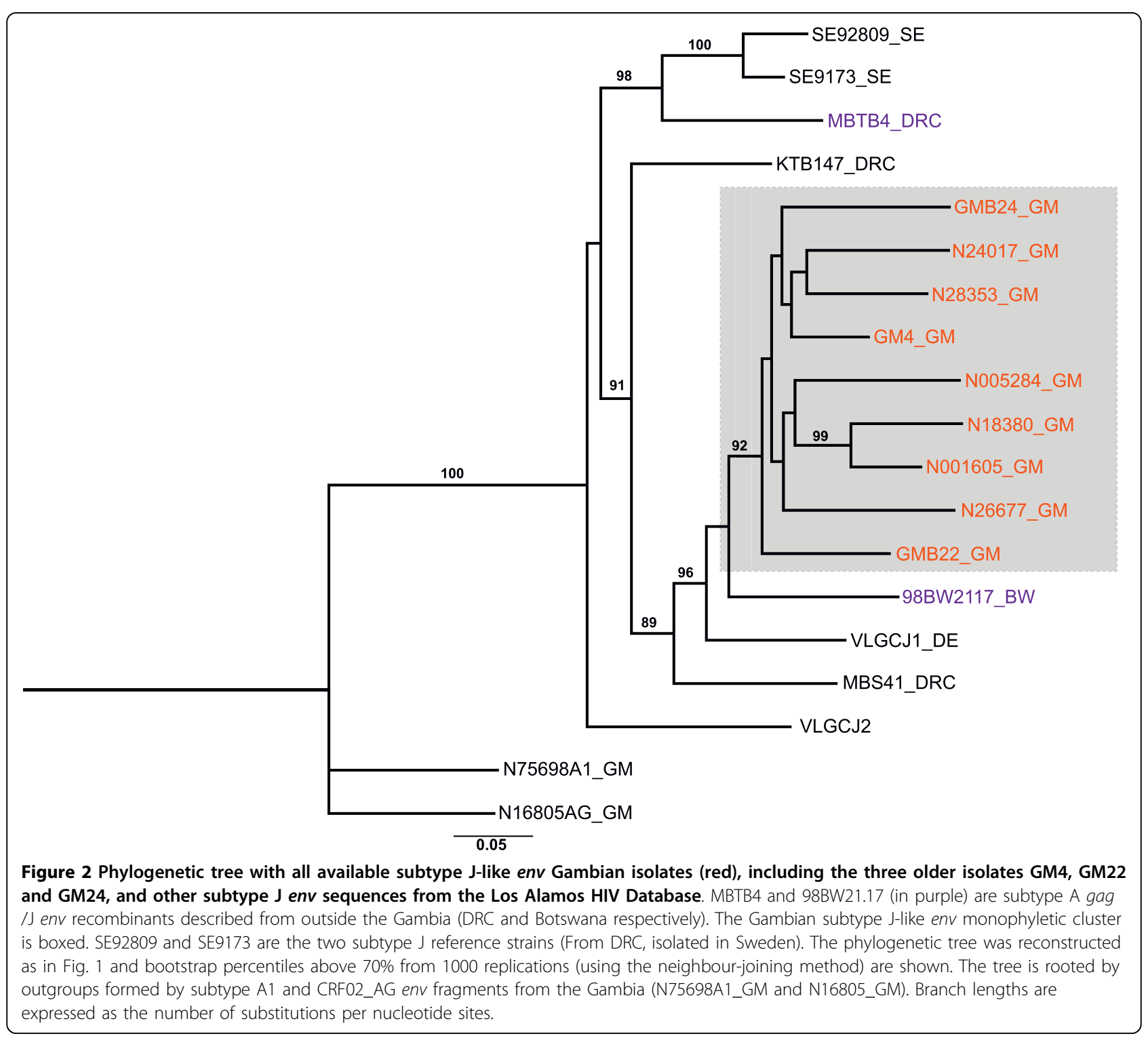


env region [31]. The subtype A gag phylogenetic tree was re-built including this isolate, along with three further DRC subtype A sequences (KCC2, KTB13 and KTB035), which required use of a shorter fragment length as described above. The MTBT4 isolate $g a g$ appears to be more closely related to subtype A gag regions from gag A/env J-like recombinants than other subtype A sequences (with a bootstrap support of 76\%), including those from DRC (Fig. 3). Of note, the env region from MTBT4 clusters with the two reference J envs SE9173 (from an individual known to be infected in DRC) and SE92809 (bootstrap support of 98), rather than the other $e n v \mathrm{~J}$ isolates with subtype A gag regions (Fig. 2).

\section{CRF49_cpx, a novel circulating recombinant form}

Near full-genome sequences from three of the gag A/env J-like isolates (N18380_GM, N28353_GM and N26677_GM) were generated and a phylogenetic tree constructed as described above (Fig. 4), which provided confirmation that these viruses represent a novel CRF, now named CRF49_cpx in the LAHDB. The three isolates clearly form a new cluster, separate from any currently known pure subtypes or recombinants (with a posterior probability of 1) and appear to be closely related to the Botswanan isolate 98BW21.17. Analyses of subtype recombination (as described above) revealed a complex, but consistent pattern across the three isolates (see Figs. 5, S1 and S2). In addition to the largely 


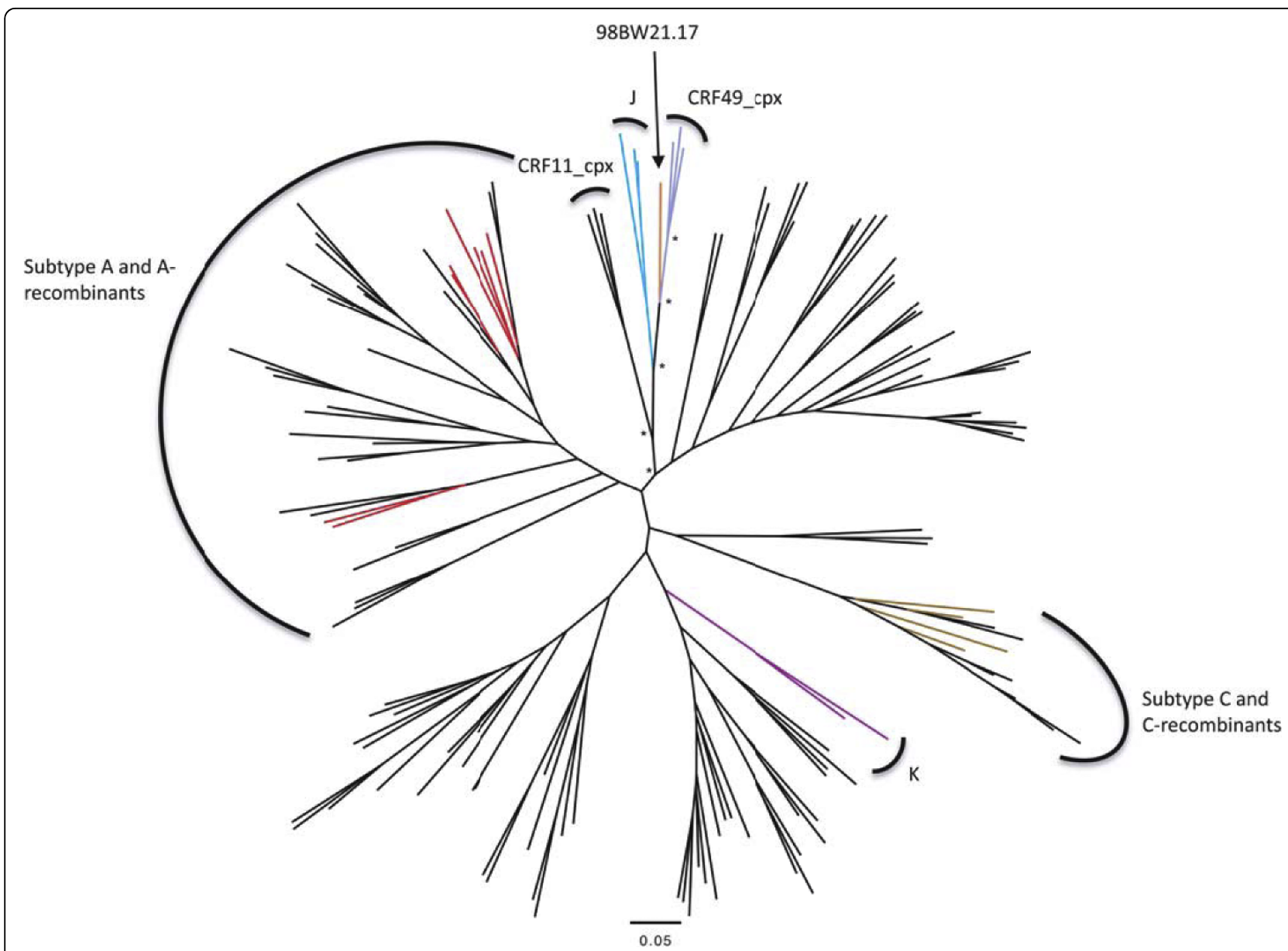

Figure 4 Midpoint rooted Bayesian tree using Los Alamos 2008 subtype reference set HIV-1 full genomes, additional A3 sequences, 98BW21.17 and 3 new Gambian CRF49_cpx isolates. Pure subtype sequences represented in the new Gambian complex recombinant are shown in color (A (red), J (turquoise), C (brown), K (purple)). Relevant nodes to the new complex recombinant, with a posterior probability of 1 , are marked with *

subtype A gag region and J-like env, a significant subtype $\mathrm{C}$ fragment is present in a portion of pol, extending through vif to $v p r$ (which is absent in 98BW21.17), where a breakpoint with the subtype J-like fragment is found. The pol gene is mosaic and contains regions with similarity to subtypes $\mathrm{A}, \mathrm{J}, \mathrm{K}$ and $\mathrm{C}$, as well a fragment which is not clearly defined by currently known pure subtype sequences. A phylogenetic tree constructed with this pol fragment (not resolved through Simplot bootscanning analysis), suggested that this region was subtype F-like (Fig. 5). Simplot and bootscan analysis [22] clearly showed a similar pattern of subtype recombination across the three isolates, although there was variation in where the exact breakpoints are (Supplementary Fig. S1 and S2), especially in the highly mosaic pol gene. The diversity between the three CRF49_cpx sequences may suggest that they are derived from a virus that recombined decades ago and as a great deal of evolution may have occurred since that time, many of the recombination breakpoints cannot be clearly defined. The Simplot and bootscan analysis [22] was repeated for each sequence, with inclusion of the Botswanan isolate 98BW21.17 in the reference set. This suggested that apart from the subtype C-like fragment, the CRF49_cpx sequences are more similar to $98 \mathrm{BW} 21.17$ than to most pure reference subtypes representing each recombinant fragment (Supplementary Fig. S3). It is possible, therefore, that CRF49_cpx originated via further recombination between a 98BW21.17-like strain and a subtype C isolate.

A careful examination of patient records was performed to determine social factors that might be associated with the CRF49_cpx viruses. There was no evidence that any of these subjects were related and there was no exclusive association with an ethnic group in this set of subjects (two Mandinka, two Manjago, one Jola and one Serahuli - see Table 1). None of these subjects were reported commercial sex workers (CSWs), 


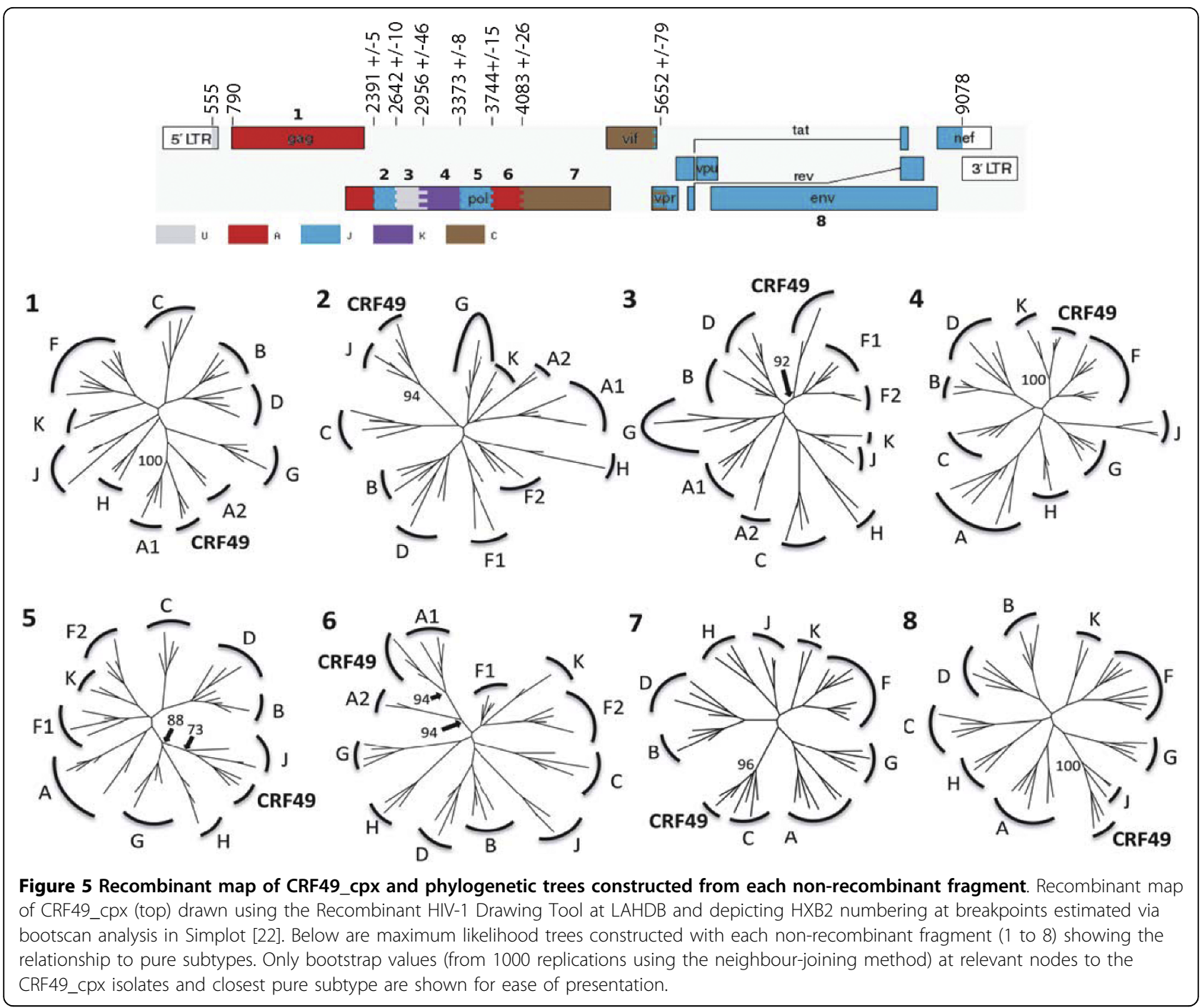

one reported a blood transfusion and there were no reports of travel to the DRC or Botswana for any of the patients.

\section{HIV-1 subtype distribution relative to Senegal}

The most recent survey from Senegal show a high prevalence of subtype C (40\%), followed by CRF 02_AG (24.3\%), then subtype B (18.6\%) in a Senegalese cohort of men who have sex with men [32]. This distribution was different from female sex workers (FSWs) and from the general population where CRF02_AG was reported to predominate [33]. In the Senegalese FSW cohort, despite large sample numbers (328), only 2 subtype J isolates (in env) were reported. Because a small (385 bp) C2-V3 env fragment was used for subtyping [32,33], there is a concern that this might have missed detecting subtypes Js. However when the Gambian 38 samples plus the Los Alamos reference set are trimmed to the 385 bp C2-V3 region used in the Senegalese study, the six new Gambian subtype J-like env sequences still cluster with the reference J sequences with high bootstrap values (results not shown). If J subtypes or CRF49_cpx isolates were present in the Senegalese cohort, they would have been detected by the 385 bp C2-V3 analysis, therefore the high frequency of CRF49_cpx isolates observed in the Gambia may not extend to neighboring Senegal.

The geographical and subtype information in the LAHDB are gathered from investigator-supplied information. Different levels of rigor can be used to define HIV-1 subtype (e.g. the REGA HIV subtyping algorithm [34] requires a minimum of $800 \mathrm{bp}$ of sequence whereas many of the LAHDB subtype designations are provided for sequences of less than $300 \mathrm{bp}$ ). Furthermore, of subtype designations, there can be multiple listings for the same patient and this may result in over-reporting of some subtypes. For example, for CRF02_AG, when the 840 Senegalese entries in LAHDB with reported subtype 
are screened for entries $800 \mathrm{bp}$ or larger and the known multiple patient entries are removed, a set of 183 sequence entries remain. These 183 sequences were analysed phylogenetically, using maximum likelihood methods as described above, to generate a more stringent subtype distribution (Fig. 6, left pie). Similar criteria were applied to the 38 novel Gambian sequences from this study plus the four Gambian LAHDB entries $>800$ bp (Fig. 6, right pie). In this analysis, there are large differences in the frequency of the HIV-1 subtypes between the two countries (Fig 6). This could be due to cultural differences, or to differences in the age and extent of the epidemic in each country. In addition, the Senegalese data are dominated by sequences derived from specific cohorts (MSM, CSW) while the Gambia data (mostly derived from the current study) come from random selection of patients attending a GUM clinic; such differences in the patient composition could results in the large differences in the subtype distribution.

\section{Conclusions}

Information on the diversity of HIV-1 in the Gambia is currently lacking and the current study has attempted to address this gap by generating full-length HIV-1 env sequences from 38 local HIV-1 isolates. Documentation of the ongoing HIV-1 epidemic and sequence data from West Africa is important for several reasons. In a region where HIV-1 diversity is higher than in many other parts of sub-Saharan Africa, such information is required to maintain accurate viral diagnostics and sensitive viral load assays. HIV-1 subtypes may differ biologically in areas such as viral fitness $[35,36]$ and co-receptor usage (e.g. likelihood of switch from R5 to $\mathrm{X} 4$ usage) $[37,38]$. These may in turn translate into higher risk of disease progression in certain subtypes and recombinant viruses could also have certain advantages over their parent strains. Studies in East Africa, using both prevalent and incident infections, have shown a higher risk of progression to AIDS and AIDSrelated death in subtype D (and inter-subtype recombinant) -infected individuals when compared to subtype A-infected patients $[39,40]$; even following adjustment for baseline viral load [41]. A Senegalese study supports the notion that non-A subtype infections progress faster than subtype A infections [42], although outcomes in CRF02_AG infected individuals appear to be no worse compared to non-AG infections [43]; despite the rise of this circulating recombinant form (CRF) in West Africa and in vitro data suggesting enhanced viral fitness [35]. With the increasing availability of anti-retroviral therapy (ART) in West Africa, it is also important to consider potential differences between HIV-1 subtypes in drug resistance pathways and the ease with which resistance appears due to naturally occurring polymorphisms (e.g. the development of $\mathrm{K} 65 \mathrm{R}$ in subtype $\mathrm{C}$ infections) $[44,45]$. Such findings would clearly have implications for local ART regimes and choice of $2^{\text {nd }}$ line drugs. Finally, local sequence data are important in the design of potential immunogens for future prophylactic and therapeutic HIV-1 vaccines, although the greater

\section{Senegal}

\section{Gambia}
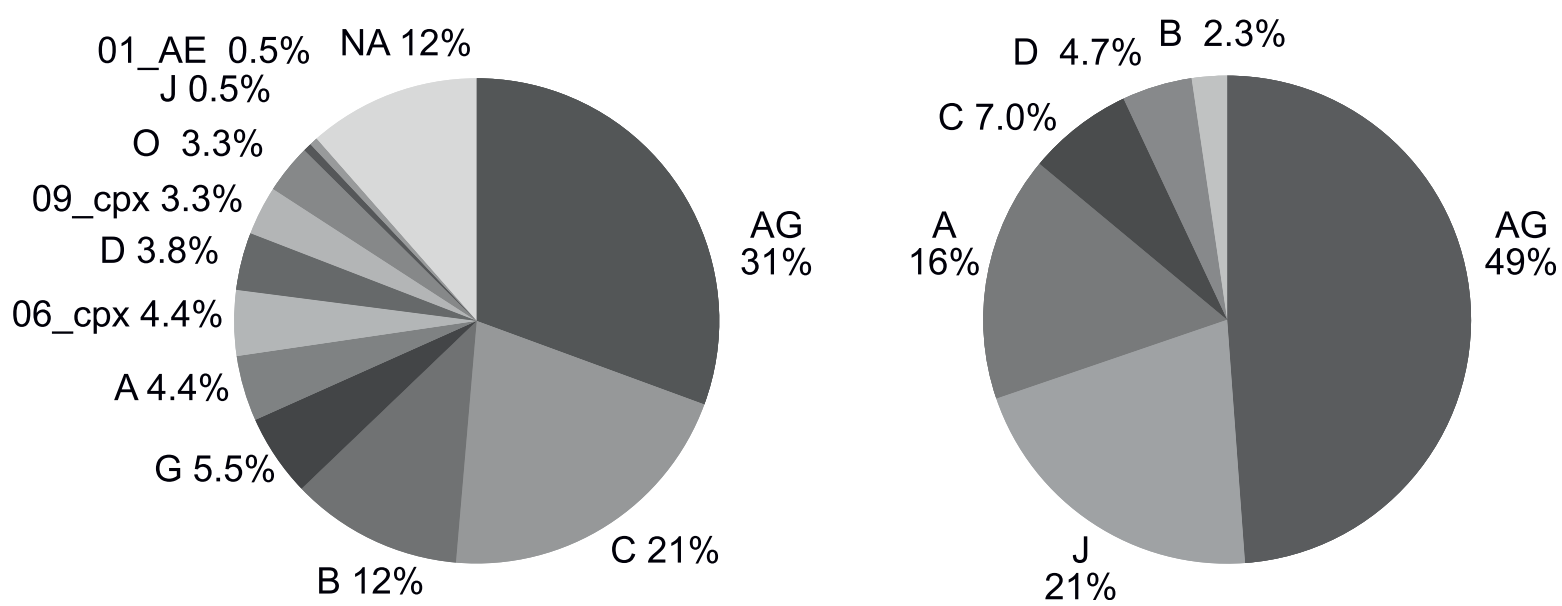

Figure 6 HIV-1 subtype distribution in Senegal compared to Gambia. The left chart shows the distribution in the 183 LAHDB sequences from Senegal $>800 \mathrm{bp}$. The right chart shows the distribution in all LAHDB subtyped HIV-1 sequences from Gambia $>800$ bp (4 entries) plus the 38 sequences from the current work (42 entries total). Note that the CRF49_cpx viruses identified in this study were included in the J category. The frequency of selected subtypes with 95\% confidence intervals (calculated using the modified Wald method) were Gambia J 21\% (11.235.4\%), Gambia CRF 02_AG 49\% (34.6-63.3\%); Senegal J 0.6\% (< 0.01 to 3.3\%), Senegal CRF 02_AG 31\% (24-38\%). 
diversity in West Africa makes this daunting task even more challenging in this subregion. Mosaic vaccine strategies $[4,5]$ may overcome this barrier and documentation of new CRFs and accurate representation of global sequence diversity is essential for these strategies.

While the majority prevalence of subtype A and CRF02_AG in the new set of HIV-1 isolates is consistent with data from other West African countries, the identification of 6 isolates of a novel recombinant, CRF49_cpx, in the 38 isolates was surprising and unique to the Gambia. These six infected individuals were epidemiologically unlinked and env sequence from these viruses cluster with three previously described Gambian subtype J-like env sequences. Thus, all nine isolates are likely to represent the novel HIV-1 CRF49_cpx. Full genome sequence from the Botswanan isolate (98BW21.17) [30] is closely linked to the Gambian isolates in phylogenetic analyses (more so than to any other virus currently in the LAHDB). Due to the limited number of patients examined, it is difficult to predict the importance of CRF49_cpx in the Gambian HIV-1 epidemic. Although some criteria were imposed in sample selection, within both patient groups $(\mathrm{CD} 4>/=$ $28 \%$ at first presentation and recently diagnosed and commenced antiretroviral therapy) selection was randomized. There is good reason to believe therefore, that CRF49_cpx may represent a reasonable proportion of the HIV-1 infections in the Gambia. Further studies are important to clarify its prevalence (including changes over time), the contribution to new infections in recent years and the disease potential relative to other local subtypes.

\section{Additional material}

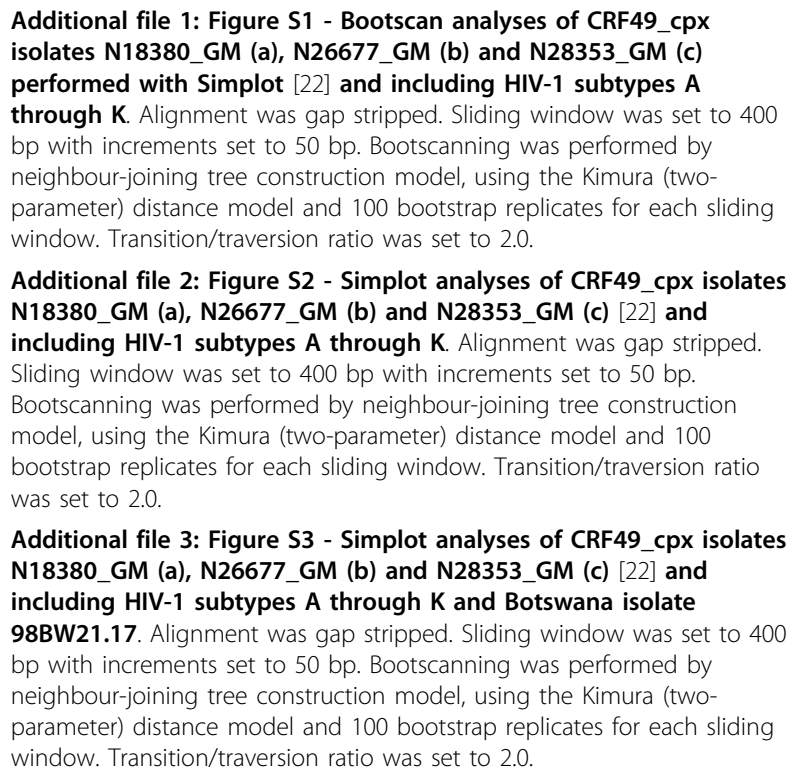

\section{Acknowledgements}

TdS is supported by a Medical Research Council (UK) Clinical Research Training Fellowship. We would like to thank the patients and staff at the MRC (UK) Fajara Genitourinary Medicine (GUM) clinic for their participation in the HIV cohort, which made this study possible.

\section{Author details}

${ }^{1}$ Medical Research Council (UK) Laboratories, Atlantic Road, PO Box 273, Fajara, The Gambia. ${ }^{2} \mathrm{MRC} / \mathrm{UCL}$ Centre for Medical Molecular Virology, Division of Infection and Immunity, University College London, UK. ${ }^{3}$ Weatherall Institute of Molecular Medicine, Medical Research Council Human Immunology Unit, John Radcliffe Hospital, Oxford, UK. ${ }^{4}$ Theoretical Biology \& Biophysics, Los Alamos National Laboratory, Los Alamos, NM 87545, USA.

\section{Authors' contributions}

TdS participated in all parts of the study, co-supervised the work, was responsible for env and gag sequencing, participated in the sequence and phylogenetic analysis and participated in writing the manuscript, RT was responsible for amplifying and sequencing most of the envs and helped with the initial analysis of the sequences, SH provided detailed phylogenetic support and assisted in writing the manuscript, RTr assisted in generating near full-genome sequence from the three described CRF49_cpx isolates, CVT provided database analysis, organized the patient data and participated in writing the manuscript, $\mathrm{CO}$ participated in designing the sequencing strategy and methods and assisted in the initial analysis of the sequences, BF helped interpret the recombination and phylogenetic analyses of CRF49_cpx and assisted in writing the manuscript, HW participated in the design of the study, provided virological support and participated in writing the manuscript, SR-J participated in the design of the study, provided virological support and participated in writing the manuscript, AJ provided virological support and participated in writing the manuscript, $M C$ participated in all parts of the study, co-supervised the work, assisted in the sequence analysis and phylogenetic analysis and participated in writing the manuscript.

\section{Competing interests}

The authors declare that they have no competing interests.

Received: 21 April 2010 Accepted: 9 October 2010

Published: 9 October 2010

\section{References}

1. Schim van der Loeff MF, Sarge-Njie R, Ceesay $S$, Awasana AA, Jaye $P$, Sam O, Jaiteh KO, Cubitt D, Milligan P, Whittle HC: Regional differences in HIV trends in The Gambia: results from sentinel surveillance among pregnant women. Aids 2003, 17:1841-1846.

2. van der Loeff MF, Awasana AA, Sarge-Njie R, van der Sande M, Jaye A Sabally S, Corrah T, McConkey SJ, Whittle HC: Sixteen years of HIV surveillance in a West African research clinic reveals divergent epidemic trends of HIV-1 and HIV-2. Int J Epidemiol 2006, 35:1322-1328.

3. Los Alamos HIV Database. [http://www.hiv.lanl.gov/].

4. Barouch DH, O'Brien KL, Simmons NL, King SL, Abbink P, Maxfield LF, Sun YH, La Porte A, Riggs AM, Lynch DM, et al: Mosaic HIV-1 vaccines expand the breadth and depth of cellular immune responses in rhesus monkeys. Nat Med 16:319-323.

5. Santra S, Liao HX, Zhang R, Muldoon M, Watson S, Fischer W, Theiler J, Szinger J, Balachandran $H$, Buzby A, et al: Mosaic vaccines elicit CD8+ T lymphocyte responses that confer enhanced immune coverage of diverse HIV strains in monkeys. Nat Med 16:324-328.

6. Salazar-Gonzalez JF, Bailes E, Pham KT, Salazar MG, Guffey MB, Keele BF, Derdeyn CA, Farmer P, Hunter E, Allen S, et al: Deciphering human immunodeficiency virus type 1 transmission and early envelope diversification by single-genome amplification and sequencing. J Virol 2008, 82:3952-3970.

7. Metabion. [http://www.metabion.com/].

8. Macrogen. [http://www.macrogen.com/].

9. Hall TA: BioEdit: a user-friendly biological sequence alignment editor and analysis program for Windows 95/98/NT. Nucleic Acids Symposium Series 1999, 41:95-98. 
10. BioEdit. [http://www.mbio.ncsu.edu/bioedit/bioedit.html].

11. Stothard P: The sequence manipulation suite: JavaScript programs for analyzing and formatting protein and DNA sequences. Biotechniques 2000, 28:1102, 1104.

12. Sequence Manipulation Suite. [http://www.bioinformatics.org/sms2/ orf_find.html].

13. Meloni ST, Kim B, Sankale JL, Hamel DJ, Tovanabutra S, Mboup S, McCutchan FE, Kanki PJ: Distinct human immunodeficiency virus type 1 subtype A virus circulating in West Africa: sub-subtype A3. J Virol 2004, 78:12438-12445.

14. Meloni ST, Sankale JL, Hamel DJ, Eisen G, Gueye-Ndiaye A, Mboup S, Kanki PJ: Molecular epidemiology of human immunodeficiency virus type 1 sub-subtype A3 in Senegal from 1988 to 2001. J Virol 2004, 78:12455-12461.

15. Larkin MA, Blackshields G, Brown NP, Chenna R, McGettigan PA, McWilliam H, Valentin F, Wallace IM, Wilm A, Lopez R, et al: Clustal W and Clustal $\times$ version 2.0. Bioinformatics 2007, 23:2947-2948.

16. EBI ClustalW2. [http://www.ebi.ac.uk/Tools/clustalw2/index.html].

17. Phylogenetic analysis using parsimony and other methods. Version 4.0b10. [http://paup.csit.fsu.edu/paupfaq/faq.html].

18. Felsenstein J: Maximum-likelihood estimation of evolutionary trees from continuous characters. Am J Hum Genet 1973, 25:471-492.

19. Yang Z: Estimating the pattern of nucleotide substitution. J Mol Evol 1994, 39:105-111.

20. Inkscape. [http://www.inkscape.org/]

21. Huelsenbeck JP, Ronquist F: MRBAYES: Bayesian inference of phylogenetic trees. Bioinformatics 2001, 17:754-755.

22. Lole KS, Bollinger RC, Paranjape RS, Gadkari D, Kulkarni SS, Novak NG, Ingersoll R, Sheppard HW, Ray SC: Full-length human immunodeficiency virus type 1 genomes from subtype C-infected seroconverters in India, with evidence of intersubtype recombination. J Virol 1999, 73:152-160

23. Gambian 2003 Census Data. [https://www.cia.gov/library/publications/theworld-factbook/fields/2075.html].

24. Carr JK, Salminen MO, Albert J, Sanders-Buell E, Gotte D, Birx DL, McCutchan FE: Full genome sequences of human immunodeficiency virus type 1 subtypes $G$ and $A / G$ intersubtype recombinants. Virology 1998, 247:22-31.

25. Abecasis $A B$, Lemey $P$, Vidal $N$, de Oliveira T, Peeters $M$, Camacho $R$, Shapiro B, Rambaut A, Vandamme AM: Recombination confounds the early evolutionary history of human immunodeficiency virus type 1 : subtype $\mathrm{G}$ is a circulating recombinant form. J Virol 2007, 81:8543-8551.

26. Zhang M, Foley B, Schultz AK, Macke JP, Bulla I, Stanke M, Morgenstern B, Korber $B$, Leitner T: The role of recombination in the emergence of a complex and dynamic HIV epidemic. Retrovirology 7:25.

27. Bobkov A, Cheingsong-Popov R, Salminen M, McCutchan F, Louwagie J, Ariyoshi $\mathrm{K}$, Whittle $\mathrm{H}$, Weber J: Complex mosaic structure of the partial envelope sequence from a Gambian HIV type 1 isolate. AIDS Res Hum Retroviruses 1996, 12:169-171.

28. Cham F, Heyndrickx L, Janssens W, Van der Auwera G, Vereecken K, De Houwer K, Coppens S, Whittle H, van der Groen G: Study of HIV type 1 gag/env variability in The Gambia, using a multiplex DNA polymerase chain reaction. AIDS Res Hum Retroviruses 2000, 16:1915-1919.

29. Binley JM, Wrin T, Korber B, Zwick MB, Wang M, Chappey C, Stiegler G, Kunert R, Zolla-Pazner $\mathrm{S}$, Katinger $\mathrm{H}$, et al: Comprehensive cross-clade neutralization analysis of a panel of anti-human immunodeficiency virus type 1 monoclonal antibodies. J Virol 2004, 78:13232-13252.

30. Novitsky VA, Gaolekwe S, McLane MF, Ndung'u TP, Foley BT, Vannberg F, Marlink R, Essex M: HIV type $1 \mathrm{~A} / \mathrm{J}$ recombinant with a pronounced pol gene mosaicism. AIDS Res Hum Retroviruses 2000, 16:1015-1020.

31. Vidal N, Peeters M, Mulanga-Kabeya C, Nzilambi N, Robertson D, llunga W, Sema H, Tshimanga K, Bongo B, Delaporte E: Unprecedented degree of human immunodeficiency virus type 1 (HIV-1) group M genetic diversity in the Democratic Republic of Congo suggests that the HIV-1 pandemic originated in Central Africa. J Virol 2000, 74:10498-10507.

32. Ndiaye HD, Kane CT, Vidal N, Niama FR, Niang-Diallo PA, Dieye T, GayeDiallo A, Wade AS, Peeters M, Mboup S: Surprisingly High Prevalence of Subtype $C$ and Specific HIV-1 Subtype/CRF Distribution in Men Having Sex With Men in Senegal. J Acquir Immune Defic Syndr 2009.

33. Hamel DJ, Sankale JL, Eisen G, Meloni ST, Mullins C, Gueye-Ndiaye A, Mboup S, Kanki PJ: Twenty years of prospective molecular epidemiology in Senegal: changes in HIV diversity. AIDS Res Hum Retroviruses 2007, 23:1189-1196.

34. Abecasis $A B$, Wang $Y$, Libin $P$, Imbrechts $S$, de Oliveira $T$, Camacho RJ, Vandamme AM: Comparative performance of the REGA subtyping tool version 2 versus version 1. Infect Genet Evol 10:380-385.

35. Njai HF, Gali Y, Vanham G, Clybergh C, Jennes W, Vidal N, Butel C, MpoudiNgolle E, Peeters M, Arien KK: The predominance of Human Immunodeficiency Virus type 1 (HIV-1) circulating recombinant form 02 (CRF02_AG) in West Central Africa may be related to its replicative fitness. Retrovirology 2006, 3:40.

36. Abraha A, Nankya IL, Gibson R, Demers K, Tebit DM, Johnston E, Katzenstein D, Siddiqui A, Herrera C, Fischetti L, et al: CCR5- and CXCR4tropic subtype $C$ human immunodeficiency virus type 1 isolates have a lower level of pathogenic fitness than other dominant group $\mathrm{M}$ subtypes: implications for the epidemic. J Virol 2009, 83:5592-5605.

37. Tscherning C, Alaeus A, Fredriksson R, Bjorndal A, Deng H, Littman DR, Fenyo EM, Albert J: Differences in chemokine coreceptor usage between genetic subtypes of HIV-1. Virology 1998, 241:181-188.

38. Ping LH, Nelson JA, Hoffman IF, Schock J, Lamers SL, Goodman M, Vernazza P, Kazembe P, Maida M, Zimba D, et al: Characterization of V3 sequence heterogeneity in subtype $C$ human immunodeficiency virus type 1 isolates from Malawi: underrepresentation of X4 variants. J Virol 1999, 73:6271-6281.

39. Kaleebu P, French N, Mahe C, Yirrell D, Watera C, Lyagoba F, Nakiyingi J, Rutebemberwa A, Morgan D, Weber J, et al: Effect of human immunodeficiency virus (HIV) type 1 envelope subtypes $A$ and $D$ on disease progression in a large cohort of HIV-1-positive persons in Uganda. J Infect Dis 2002, 185:1244-1250.

40. Kiwanuka N, Laeyendecker O, Robb M, Kigozi G, Arroyo M, McCutchan F, Eller LA, Eller M, Makumbi F, Birx D, et al: Effect of human immunodeficiency virus Type 1 (HIV-1) subtype on disease progression in persons from Rakai, Uganda, with incident HIV-1 infection. J Infect Dis 2008, 197:707-713.

41. Baeten JM, Chohan B, Lavreys L, Chohan V, McClelland RS, Certain L, Mandaliya $K$, Jaoko W, Overbaugh J: HIV-1 subtype $D$ infection is associated with faster disease progression than subtype $A$ in spite of similar plasma HIV-1 loads. J Infect Dis 2007, 195:1177-1180.

42. Kanki PJ, Hamel DJ, Sankale JL, Hsieh C, Thior I, Barin F, Woodcock SA, Gueye-Ndiaye A, Zhang E, Montano M, et al: Human immunodeficiency virus type 1 subtypes differ in disease progression. J Infect Dis 1999, 179:68-73.

43. Laurent C, Bourgeois A, Faye MA, Mougnutou R, Seydi M, Gueye M, Liegeois F, Kane CT, Butel C, Mbuagbaw J, et al: No difference in clinical progression between patients infected with the predominant human immunodeficiency virus type 1 circulating recombinant form (CRF) 02_AG strain and patients not infected with CRF02_AG, in Western and West-Central Africa: a four-year prospective multicenter study. I Infect Dis 2002, 186:486-492

44. Invernizzi CF, Coutsinos D, Oliveira M, Moisi D, Brenner BG, Wainberg MA: Signature nucleotide polymorphisms at positions 64 and 65 in reverse transcriptase favor the selection of the K65R resistance mutation in HIV1 subtype C. J Infect Dis 2009, 200:1202-1206.

45. Martinez-Cajas JL, Pai NP, Klein MB, Wainberg MA: Differences in resistance mutations among HIV-1 non-subtype B infections: a systematic review of evidence (1996-2008). J Int AIDS Soc 2009, 12:11.

doi:10.1186/1742-4690-7-82

Cite this article as: de Silva et al:: HIV-1 subtype distribution in the Gambia and the significant presence of CRF49_cpx, a novel circulating recombinant form. Retrovirology 2010 7:82. 